\title{
Polarizing properties and structure of the cuticle of scarab beetles from the Chrysina genus
}

\author{
Lía Fernández del Río, Hans Arwin, and Kenneth Järrendahl \\ Laboratory of Applied Optics, Department of Physics, Chemistry and Biology, Linköping University, SE-581 83 Linköping, Sweden
}

(Received 13 January 2016; published 18 July 2016)

\begin{abstract}
The optical properties of several scarab beetles have been previously studied but few attempts have been made to compare beetles in the same genus. To determine whether there is any relation between specimens of the same genus, we have studied and classified seven species from the Chrysina genus. The polarization properties were analyzed with Mueller-matrix spectroscopic ellipsometry and the structural characteristics with optical microscopy and scanning electron microscopy. Most of the Chrysina beetles are green colored or have a metallic look (gold or silver). The results show that the green-colored beetles polarize reflected light mainly at off-specular angles. The gold-colored beetles polarize light left-handed near circular at specular reflection. The structure of the exoskeleton is a stack of layers that form a cusplike structure in the green beetles whereas the layers are parallel to the surface in the case of the gold-colored beetles. The beetle C. gloriosa is green with gold-colored stripes along the elytras and exhibits both types of effects. The results indicate that Chrysina beetles can be classified according to these two major polarization properties.
\end{abstract}

DOI: 10.1103/PhysRevE.94.012409

\section{INTRODUCTION}

The attractive shiny metallic coloration of Chrysina beetles in the Scarabaeidae family has made them highly attractive by insect collectors. They are also widely studied by researchers due to their brilliant iridescence and polarization features. Michelson noticed already in the early 1900s that light reflected from the scarab beetle Chrysina resplendens (Boucard, 1875) has a high degree of circular polarization [1]. Since then, a series of investigations has been performed in order to explain the structural origin of the color [2-8] and polarization properties [4,9-23] of these structures. Whether or not these beetles are able to detect polarized light has also been debated [24,25].

Although optical properties of many different scarabs have been studied, the reported properties have been focused on specimens from single species. However, in what respect specimens belonging to the same family, subfamily, or genus have similar polarization characteristics has not been studied.

In this work we have made extensive studies of polarization properties on several specimens exclusively from the genus Chrysina. The selected species are shown in Table I. The Chrysina genus is of particular interest since circular polarization effects have been observed in several of its many species and it was in this genus these effects were first observed. However, it should be pointed out that circular polarization effects have been observed in beetles in other subfamilies like the Cetoniinae $[16,21,26]$. Using Mueller-matrix spectroscopic ellipsometry (MMSE) [27] in combination with structural characterization by optical microscopy and cross-sectional scanning electron microscopy (SEM) we find that, even if there is a variation in the visual coloration and patterning of the beetles, it is possible to classify the Chrysina beetles according to two major types of polarization responses connected to two different exocuticle structures. One type is the green-colored beetles $C$. woodi, C. macropus, and $C$. peruviana and the green areas of $C$. gloriosa. The other category is the metallic-colored beetles $C$. chrysargyrea, $C$. argenteola, and $C$. resplendens and the metallic-colored areas of $C$. gloriosa. The results can be

\footnotetext{
*liafe@ifm.liu.se
}

used to aid the classification of new specimens in Chrysina as well as related genera.

\section{EXPERIMENTAL DETAILS}

Scarab beetles from the Chrysina genus are found in the southern United States, Mexico, Central America, and farther south to Ecuador. The specimens of $C$. macropus, $C$. peruviana, $C$. argenteola, and $C$. resplendens used in this work were obtained as loans from different museums. On these beetles we could not use destructive techniques and, thus, only polarization characteristics were obtained. However, $C$. woodi, C. gloriosa, and C. chrysargyrea were kindly provided by Dr. P. Brady at the University of Texas at Austin, making it possible to do additional structural studies of the exoskeletons of specimens from these species.

Initial tests with left-circular and right-circular plastic sheet polarizers were performed to verify that the beetles had exoskeletons reflecting light with a high degree of circular polarization. The polarizers were examined with MMSE and found to have an extinction ratio of 2000 or better for green light, whereas at the blue and red end of the spectrum of visual light it decreased to around 20 . Next, detailed studies of reflection properties of the cuticle were performed using MMSE. A dual rotating-compensator ellipsometer (RC2, J.A. Woollam Co., Inc.) was used to record the normalized Mueller matrix of the exoskeleton of each beetle. A Mueller matrix provides a complete description of the specular reflection properties, including polarization changes and changes in degree of polarization. Several quantitative measures on polarization changes can thereby be derived as described below. Most of the measurements were performed on the scutellum, a small triangular area between the head and the wing cover, which in general is the flattest area on the exoskeleton. On C. gloriosa measurements were performed on the elytras, i.e., the wing covers. These are green with gold-colored stripes along them. Both the green and gold-colored areas were studied.

Measurements were done in the spectral range 245$1690 \mathrm{~nm}$ but only data in the range $245-1000 \mathrm{~nm}$ were considered for the analysis due to an increase of the noise level above $1000 \mathrm{~nm}$. The use of focusing lenses allowed us to measure in areas with approximately a width of $50 \mu \mathrm{m}$. 
TABLE I. Chrysina beetles included in this work. C. adelaida was not included in the study but is discussed in the concluding remarks. The photos of the beetles are rescaled to the same size, and typical sizes are given in the table.

\begin{tabular}{|c|c|c|c|c|c|}
\hline & Auctor & & & Auctor & \\
\hline Beetle name & Beetle size & Picture & Beetle name & Beetle size & Picture \\
\hline \multirow[t]{2}{*}{ C. woodi } & Horn, 1885 & & C. chrysargyrea & Sall, 1874 & \\
\hline & $3.5 \mathrm{~cm}$ & & & $3.1 \mathrm{~cm}$ & \\
\hline \multirow[t]{2}{*}{ C. macropus } & Francillon, 1795 & & C. argenteola & Bates, 1888 & \\
\hline & $3.7 \mathrm{~cm}$ & & & $3.2 \mathrm{~cm}$ & \\
\hline \multirow[t]{2}{*}{ C. peruviana } & Kirby, 1828 & & C. resplendens & Boucard, 1875 & \\
\hline & $3.3 \mathrm{~cm}$ & & & $2.8 \mathrm{~cm}$ & \\
\hline \multirow[t]{2}{*}{ C. gloriosa } & LeConte, 1854 & & C. adelaida & Hope, 1840 & \\
\hline & $2.5 \mathrm{~cm}$ & & & $3 \mathrm{~cm}$ & \\
\hline
\end{tabular}

Repeated measurements on the same spot show differences of the order of the instrument precision (typically $<0.005$ in all Mueller-matrix elements). Measurements on different positions on a cuticle and on different specimens show variations on a detailed level but the general features are the same. This is natural as beetles are individuals and cuticles are inhomogeneous, having pore canals and other irregularities. The results presented are representative data from single measurements.

Both specularly reflected and scattered light were studied. The specular MMSE measurements were performed on all beetles at angles of incidence between $20^{\circ}$ and $75^{\circ}$ in steps of $5^{\circ}$. The scattering measurements were performed on $C$. woodi, C. gloriosa, and $C$. chrysargyrea, fixing the sample holder at an incidence angle of $45^{\circ}$. In the scattering measurements, data acquisition was performed by moving the detector $\pm 15^{\circ}$ in steps of $3^{\circ}$ from the specular reflection angle, i.e., $45^{\circ}$. The angle position of the detector thus ranged from $30^{\circ}$ to $60^{\circ}$.

The reflection properties of the beetles are analyzed using the Stokes-Mueller formalism [28]. In this formalism polarized light, unpolarized light, and partially polarized light are described with the Stokes vector $\mathbf{S}$,

$$
\mathbf{S}=\left[\begin{array}{c}
I \\
Q \\
U \\
V
\end{array}\right]=\left[\begin{array}{c}
I_{x}+I_{y} \\
I_{x}-I_{y} \\
I_{+45^{\circ}}-I_{-45^{\circ}} \\
I_{r}-I_{l}
\end{array}\right],
$$

where $I_{x}, I_{y}, I_{+45^{\circ}}$, and $I_{-45^{\circ}}$ are the irradiances for linear polarization in the $x, y,+45^{\circ}$, and $-45^{\circ}$ directions, respectively, and $I_{r}$ and $I_{l}$ are the irradiances of the right-circular and the left-circular states of polarization, respectively.

The reflection properties of a beetle are described with a $4 \times 4$ Mueller matrix with elements $M_{i j}(i, j=1,2,3,4)$. We normalized $\mathbf{M}$ with $M_{11}$, the reflectance for unpolarized light, and the elements are thus defined as $m_{i j}=M_{i j} / M_{11}$. We also limit the discussion to incident unpolarized light given by $\mathbf{S}_{\mathbf{i}}=\left[I_{i}, Q_{i}, U_{i}, V_{i}\right]^{T}=[1,0,0,0]^{T}$, where we also normalized by setting $I_{i}=1$ and $\mathrm{T}$ indicates transpose.

The Stokes vector $\mathbf{S}_{\mathbf{0}}$ for the reflected beam can now be expressed as a product of $\mathbf{S}_{\mathbf{i}}$ and $\mathbf{M}$ according to $\mathbf{S}_{\mathbf{o}}=\mathbf{M} \mathbf{S}_{\mathbf{i}}$ 
and we get the following expression:

$$
\mathbf{S}_{\mathbf{o}}=\left[\begin{array}{c}
I_{o} \\
Q_{o} \\
U_{o} \\
V_{o}
\end{array}\right]=\left[\begin{array}{cccc}
1 & m_{12} & m_{13} & m_{14} \\
m_{21} & m_{22} & m_{23} & m_{24} \\
m_{31} & m_{32} & m_{33} & m_{34} \\
m_{41} & m_{42} & m_{43} & m_{44}
\end{array}\right]\left[\begin{array}{c}
1 \\
0 \\
0 \\
0
\end{array}\right]=\left[\begin{array}{c}
1 \\
m_{21} \\
m_{31} \\
m_{41}
\end{array}\right] .
$$

From $\mathbf{S}_{\mathbf{o}}$ we can determine the ellipticity angle, $\varepsilon$, degree of polarization, $P$, and polarization azimuth angle, $\alpha$, of the reflected light according to [28]

$$
\begin{gathered}
\varepsilon=\frac{1}{2} \arcsin \left(\frac{V_{o}}{\sqrt{Q_{o}^{2}+U_{o}^{2}+V_{o}^{2}}}\right) \\
=\frac{1}{2} \arcsin \left(\frac{m_{41}}{\sqrt{m_{21}^{2}+m_{31}^{2}+m_{41}^{2}}}\right), \\
\alpha=\frac{1}{2} \arctan \left(\frac{U_{o}}{Q_{o}}\right)=\frac{1}{2} \arctan \left(\frac{m_{31}}{m_{21}}\right), \\
P=\frac{\sqrt{Q_{o}^{2}+U_{o}^{2}+V_{o}^{2}}}{I_{o}}=\sqrt{m_{21}^{2}+m_{31}^{2}+m_{41}^{2}} .
\end{gathered}
$$

We also introduce the degree of circular polarization defined as $P_{\text {circ }}=V_{o} / I_{o}$ which from Eq. (2) is given by $P_{\text {circ }}=m_{41}$. In the results section we present the ellipticity $e=\tan \epsilon$ instead of $\epsilon$.

The Muller-matrix data are presented as polar contour plots where the parameters are represented as a function of wavelength $\lambda \in[245,1000] \mathrm{nm}$ and angle of incidence $\theta$ of the incident beam. Since the Mueller-matrix elements are normalized, $m_{41}$ ranges from -1 to +1 . In some plots the $m_{41}$ scale is modified to range from minimum and maximum values to enhance the visibility of small features. The degree of polarization ranges from 0 to 1 , corresponding to unpolarized and completely polarized light, respectively. The ellipticity has values from -1 (left-handed circular polarization) to +1 (right-handed circular polarization).

Finally, the absolute value of the azimuth angle is in the range $0^{\circ}-90^{\circ}$, which except when $|e|=1$ (circular polarization) represents an ellipse with its major axis orientated in the plane of incidence ( $p$-polarized) when $\alpha=0^{\circ}$, and orientated perpendicular to the plane of incidence ( $s$-polarized) when $\alpha=90^{\circ}$. The scattering plots show $m_{41}, P, e$, and $|\alpha|$.

The structure of the exoskeletons of the beetles $C$. woodi, C. gloriosa, and C. chrysargyrea was observed using two different techniques: optical microscopy to look at their surfaces and SEM to look at cross sections of their elytras. The SEM used was a Leo 1550 with a Gemini field-emission column. The samples were cooled in liquid nitrogen for 10 min, cut with a razor blade, and glued to the sample holder with silver glue. Since the samples are nonconducting, a platinum coating with a total thickness of $3.6 \mathrm{~nm}$ was deposited during $20 \mathrm{~s}$ at $60 \mathrm{~mA}$ at a pressure of $5 \times 10^{-2} \mathrm{~Pa}$. The accelerating voltage was $2 \mathrm{kV}$.

\section{RESULTS AND DISCUSSION}

In this section we first show polarization features of selected beetles using simple polarizing eyeglasses. A detailed characterization with MMSE is then presented and discussed. Finally supporting findings with optical microscopy and SEM are presented.

\section{A. Observations through polarizing filters}

The photos in Table II are taken with a left-circular polarizer in front of the camera, and without polarizer and with a rightcircular polarizer in front of the camera.

Table II shows that the light green beetle $C$. woodi appears slightly green when observed through a left-circular polarizer

TABLE II. Photos of C. woodi, C. gloriosa and C. chrysargyrea taken with a left-circular (LC) or right-circular (RC) polarizer in front of the camera or without a polarizer. The photos of the beetles are rescaled to the same size, typical sizes are given in Table I.

\begin{tabular}{lll}
\hline \hline Beetle name & LC polarizer & Ro polarizer polarizer \\
C. woodi & gloriosa \\
C. chrysargyrea &
\end{tabular}



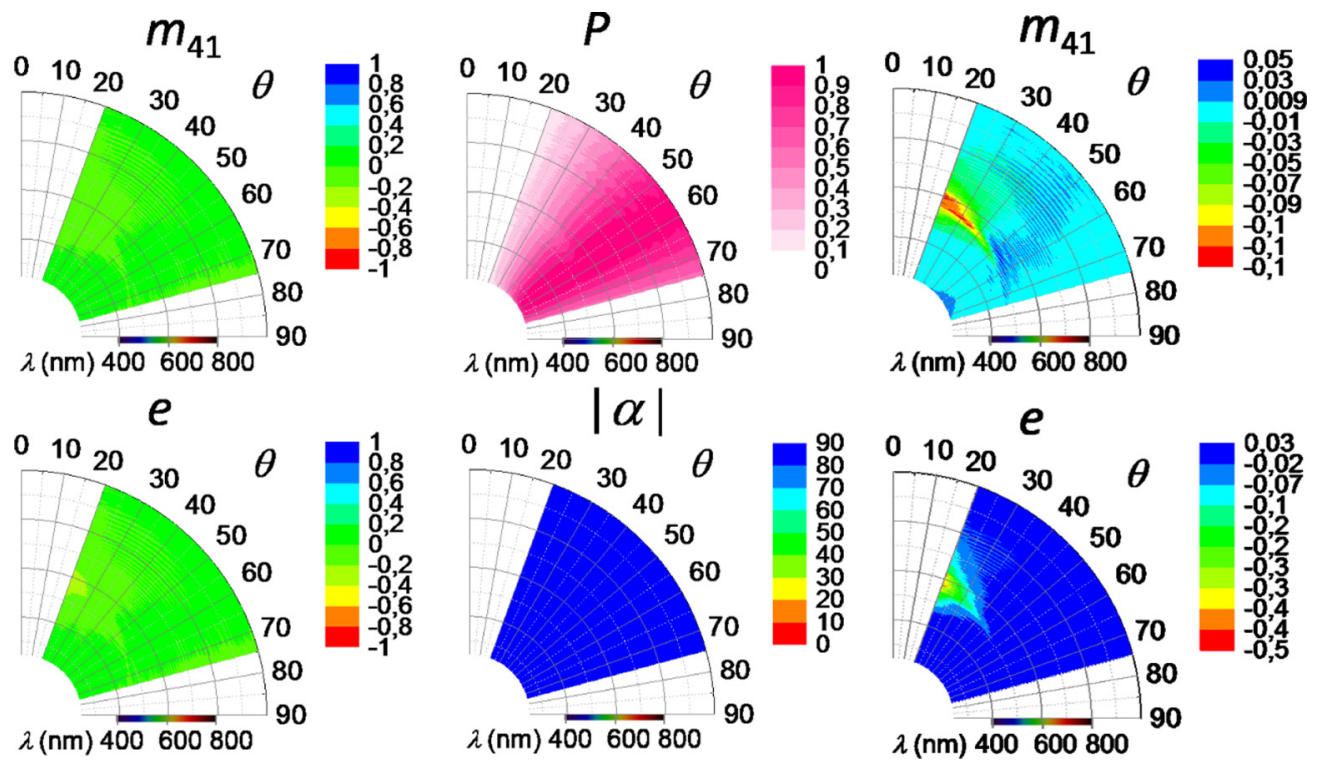

FIG. 1. Contour plots of MMSE data for $C$. woodi showing $m_{41}, P, e$, and $|\alpha|$ in the range $\lambda \in[245,1000] \mathrm{nm}$ and $\theta \in\left[20^{\circ}, 75^{\circ}\right]($ left and center). $m_{41}$ and $e$ shown rescaled to parameter maximum and minimum values (right).

but looks red-brown when observed through a right-circular polarizer. As shown in the next section, this is a combined effect of scattering of small amounts of right-handed polarized light and left-handed polarized light. The latter is observed due to the low extinction ratio of the polarizer in the red end of the spectrum. Further details are presented in the next section. Similar effects are observed for the green beetles $C$. macropus and $C$. peruviana but are not shown in Table II.

C. gloriosa is green with gold-colored stripes along its elytras. Table II indicates that $C$. gloriosa reflects only lefthanded near-circular polarized light as it becomes almost black with a right-circular polarizer. This holds for both the greenand gold-colored areas. However, right-handed polarization effects cannot be excluded based on a simple visual inspection, and a detailed MMSE analysis as presented in the next section is needed to get a better view of the polarization behavior.

Finally Table II indicates that $C$. chrysargyrea also reflects left- and right-handed near-circular polarized light which is confirmed by the MMSE in the next section. Other beetles which show similar effects are $C$. argenteola and $C$. resplendens but are not shown in Table II.

\section{B. Mueller-matrix spectroscopic ellipsometry}

Figure 1 shows results from MMSE measurements performed on $C$. woodi. Even though a clear circular polarization effect is observed by eye, the measurements show very low values on $m_{41}$, i.e., on the degree of circular polarization. Also the ellipticity is small. Figure 1 (right) shows $m_{41}$ and ellipticity on an expanded scale and it is then possible to observe some variation in the data indicating both left- and right-handed polarization but the effects are very small.

In the scattering contour plots in Fig. 2 it can be observed that even though the polarization effect is very low for specular reflection, i.e., in this case $45^{\circ}$, it increases for near-lying scattering angles, being near-circular left-handed polarized in the same wavelength range for which the weak polarization effect was found in the specular reflection as seen in Fig. 1 (right). The fact that the off-specularly scattered light is polarized explains why observation through polarizing filters with incident unpolarized light gives clear polarization effects as shown in Table II. In visual observation, the beetles C. macropus and $C$. peruviana exhibit similar polarization features as $C$. woodi and MMSE measurements confirmed that they also have similar polarization behavior.

Figure 3 shows the results of measurements on the greencolored areas of $C$. gloriosa. The MMSE data show a very low degree of circular polarization and ellipticity even though a clear circular polarization effect is observed by eye as seen in Table II. In Fig. 3 (right) $m_{41}$ and ellipticity are plotted on
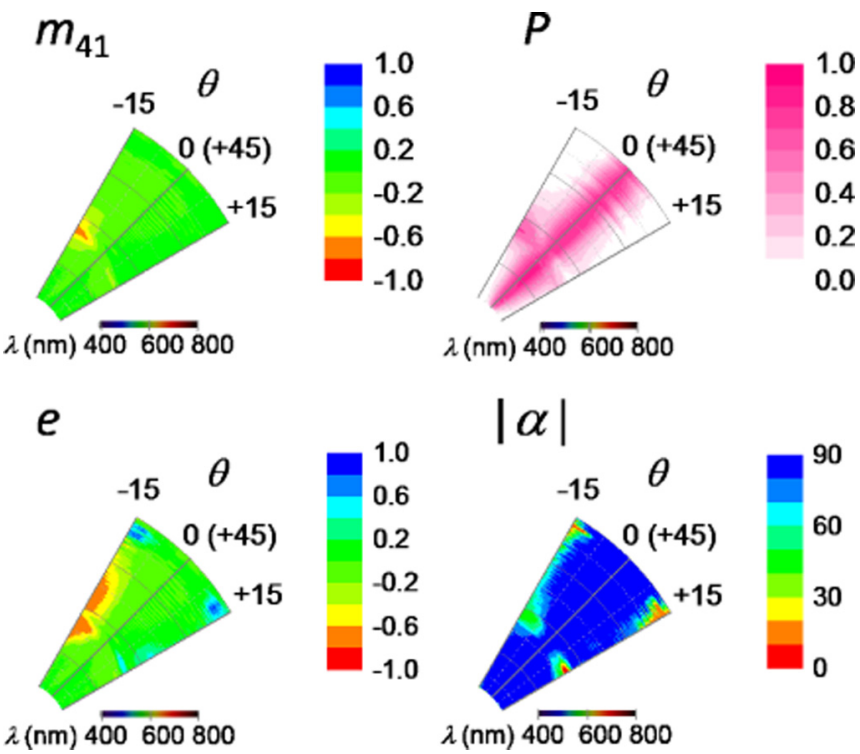

FIG. 2. Scattering contour plots of MMSE data for $C$. woodi showing $m_{41}, P, e$, and $|\alpha|$ in the range $\lambda \in[245,1000] \mathrm{nm}$ and $\theta \in\left[45^{\circ} \pm 15^{\circ}\right]$. 


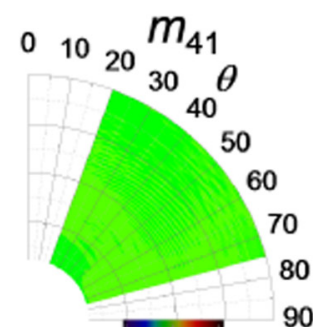

$\lambda(\mathrm{nm}) 400600800$

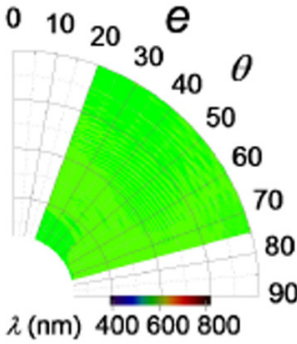

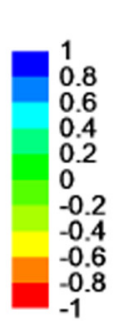

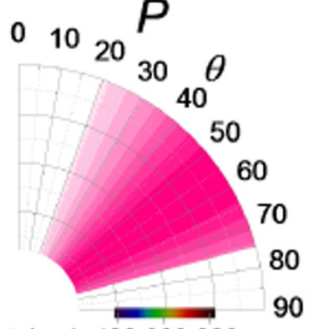

$\lambda(\mathrm{nm}) 400600800$

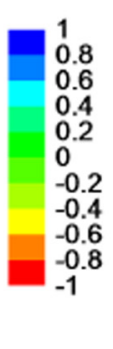

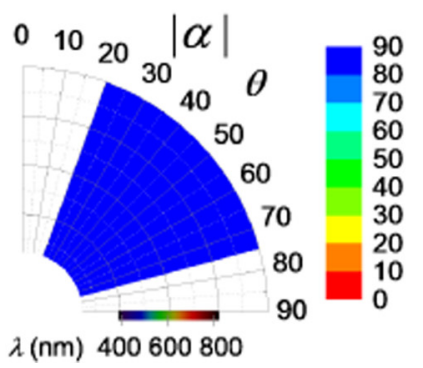
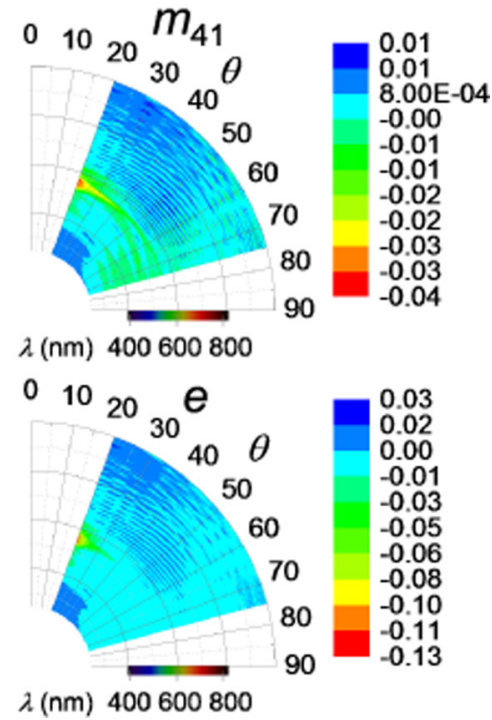

FIG. 3. Contour plots of MMSE data for $C$. gloriosa measured on a green area showing $m_{41}, P$, $e$, and $|\alpha|$ in the range $\lambda \in[245,1000] \mathrm{nm}$ and $\theta \in\left[20^{\circ}, 75^{\circ}\right]$ (left and center). $m_{41}$ and $e$ shown rescaled to parameter maximum and minimum values (right).

an expanded scale and it is possible to observe some variation in the data indicating both left- and right-handed polarization similar to $C$. woodi but the effects are slightly smaller.

The measurements on the gold-colored areas show quite different results as seen in Fig. 4. The $m_{41}$ data clearly show both left- and right-handed polarization depending on the angle of incidence and wavelength, even on a scale from -1 to 1 . Left-handed polarization can be found at angles from $20^{\circ}$ up to $75^{\circ}$ and wavelengths from 400 to $700 \mathrm{~nm}$ approximately, whereas right-handed polarization is found in a smaller range of angles, from $55^{\circ}$ to $75^{\circ}$, and wavelengths from 550 to $650 \mathrm{~nm}$. The degree of circular polarization is thus very pronounced in the gold-colored areas of the exoskeleton of $C$.
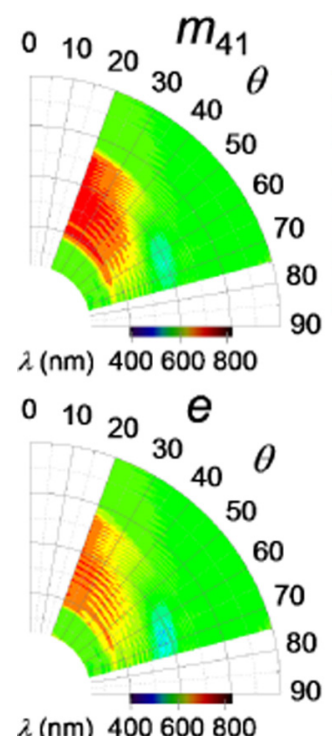
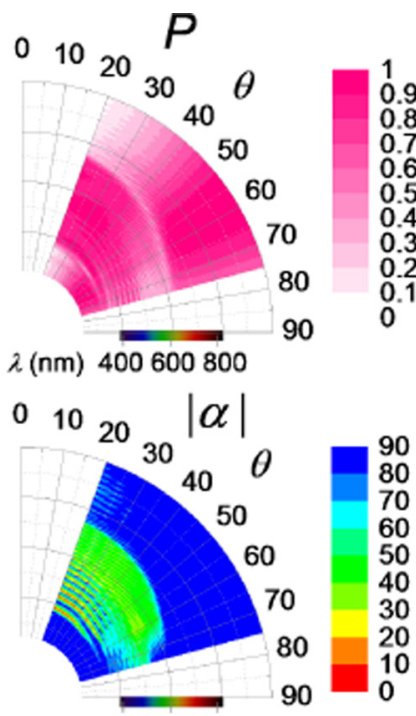

$\lambda(\mathrm{nm}) 400600800$
FIG. 4. Contour plots of MMSE data for C. gloriosa measured on a gold-colored area showing $m_{41}, P, e$, and $|\alpha|$ in the range $\lambda \in[245,1000] \mathrm{nm}$ and $\theta \in\left[20^{\circ}, 75^{\circ}\right]$. gloriosa. This is confirmed by the ellipticity plot where both high negative and positive ellipticities are reached. However, $P$ is low in the angle and wavelength regions where polarization is right-handed.

The scattering plots for C. gloriosa in Fig. 5 show different results depending on the area measured. The green-colored area shows a behavior very similar to that of $C$. woodi, $C$. peruviana, and $C$. macropus; i.e., at the specular angle, $45^{\circ}$, the polarization effect is very low whereas it increases at off-specular angles. On the other hand, the gold-colored area shows left-handed near-circular polarization at the specular angle, but this effect decreases progressively for both increasing and decreasing off-specular angles. We can also observe in Fig. 5 (right) somewhat surprisingly that the ellipticity is large and very close to -1 over a large spectral range for scattering at angles closer to the normal than the specular direction. However, as $P$ is very small in this region this effect will have very low influence on the visual appearance of the beetle.

The results of the measurements on $C$. chrysargyrea shown in Fig. 6 show a clear left-handed near-circular polarization at small incidence angles in the spectral range $\lambda=350-900 \mathrm{~nm}$ as represented by the red color in the $m_{41}$ plot. The large value on $m_{41}$ corresponds to a high degree of polarization for incident angles in the range $20^{\circ}-45^{\circ}$ and high ellipticity, close to -1 (near circular) at $\theta=20^{\circ}$ in almost the whole visible range but decreasing with $\theta$ but extending to $\theta=40^{\circ}$ or more for wavelengths from 350 to $450 \mathrm{~nm}$.

There is also a $\lambda \theta$ region with a right-handed polarization, represented by the blue region in the $m_{41}$ plot, for incidence angles in the range $55^{\circ}-65^{\circ}$ and at wavelengths from approximately 650 to $800 \mathrm{~nm}$. The ellipticity plot shows some areas where the ellipticity is getting close to 1 , but the degree of polarization is rather low.

The remaining parts of the $\lambda \theta$ region shown in the contour plots represent reflection of linear $s$-polarized light as seen from the low ellipticity and azimuth values close to $90^{\circ}$. The degree of polarization is in general high in this case. 

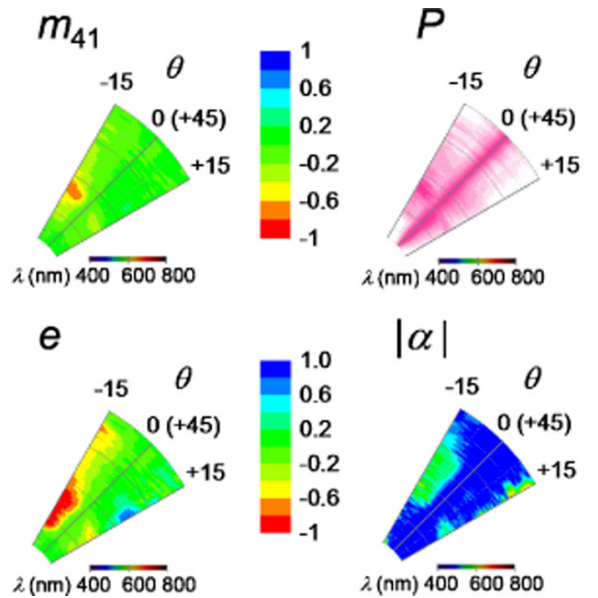
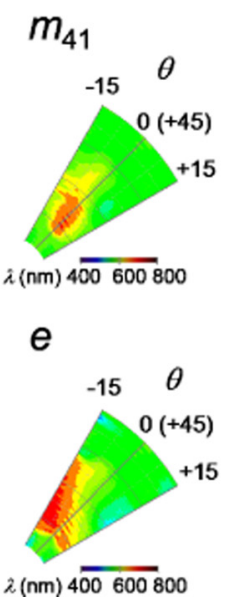
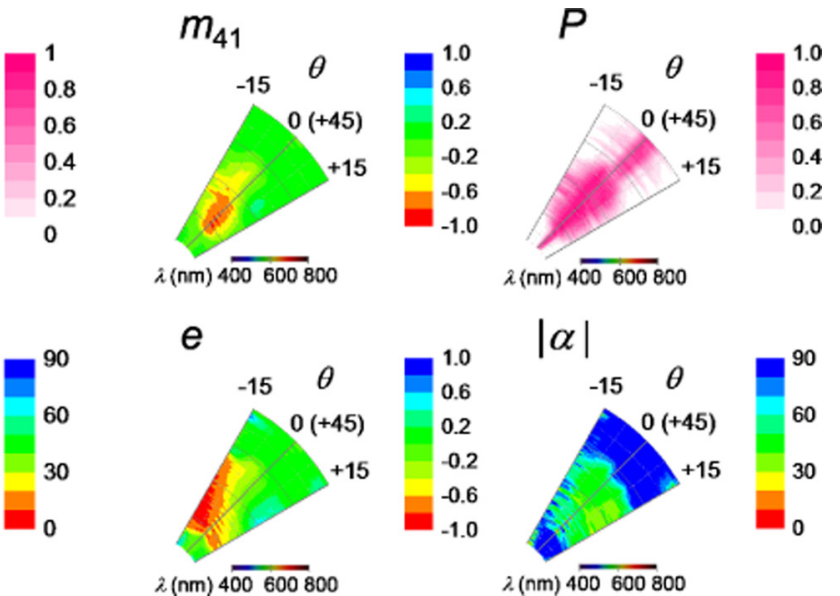

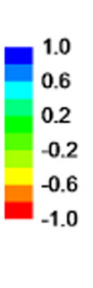

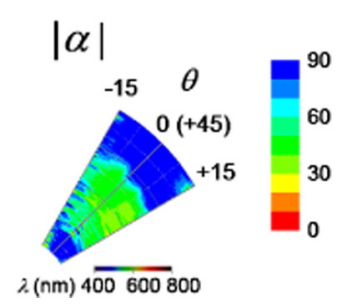

FIG. 5. Scattering contour plots of MMSE data for $C$. gloriosa showing $m_{41}, P, e$, and $|\alpha|$ in the range $\lambda \in[245,1000] \mathrm{nm}$ and $\theta \in$ $\left[45^{\circ} \pm 15^{\circ}\right]$ measured on a green area (left) and on a gold area (right).

The results shown in the scattering plots for $C$. chrysargyrea in Fig. 7 are similar to those from the gold-colored areas of C. gloriosa. At specular reflection light is left-handed nearcircularly polarized but the effect decreases for light scattered at smaller angles. The degree of polarization is, in general, very high at any angle. The ellipticity is moderate at specular reflection but increases for scattering towards the normal in contrast to the results for the $m_{41}$ element.

Specimens of $C$. argenteola and $C$. resplendens, which are silverish and goldish, respectively, have also been studied. They show a behavior similar to that of $C$. chrysargyrea with high ellipticity and high degree of polarization.

\section{Optical microscopy}

Optical microscopy images of the surface of the exoskeletons of the beetles show different structures depending on their color. In general, the green-colored beetles, C. macropus, $C$. peruviana, and $C$. woodi, and the green areas of $C$. gloriosa show a pattern composed of polygonal cells as seen in Fig. 8 . Similar structures were observed by Sharma et al. [13]. The shapes of the cells are pentagonal, hexagonal, or heptagonal, depending on the curvature of the surface, and present a depression in the center of each cell. These depressions can be distinguished by the bright yellow reflections on the images. The yellow color is due to multilayer reflection in the middle of the cells whereas the green color is caused by scattered light from the cell sides. The width of each cell is approximately $10 \mu \mathrm{m}$ as seen in Figs. 8(d) and 8(f). On the contrary, the beetles with metallic-looking coloration such as $C$. argenteola, C. chrysargyrea, and C. resplendens and the gold-colored areas of $C$. gloriosa have a very smooth surface as can be seen in Figs. 8(a), 8(b), 8(e), and 8(g).
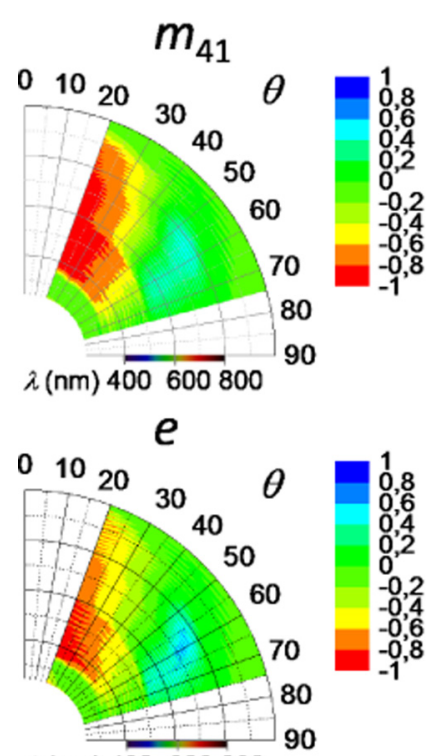

$\lambda(\mathrm{nm}) 400600800$

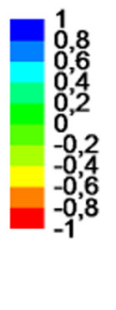

FIG. 6. Contour plots of MMSE data for C. chrysargyrea showing $m_{41}, P, e$, and $|\alpha|$ in the range $\lambda \in[245,1000] \mathrm{nm}$ and $\theta \in\left[20^{\circ}, 75^{\circ}\right]$.

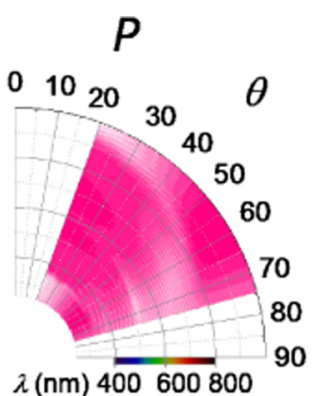

$|\alpha|$
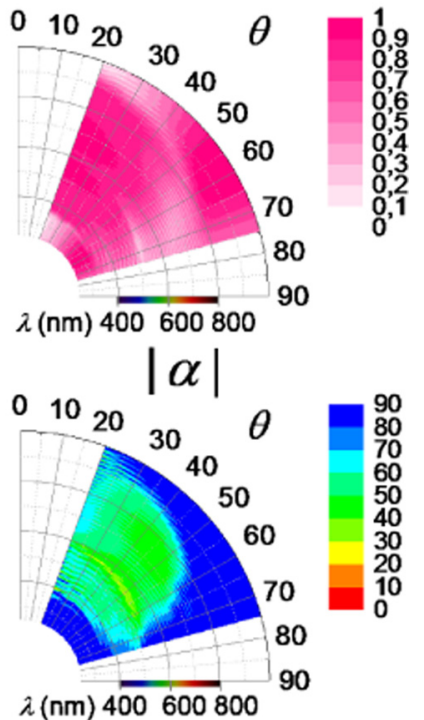

$\lambda ( \mathrm { nm } ) \longdiv { 4 0 0 6 0 0 8 0 0 }$ $m_{41}$

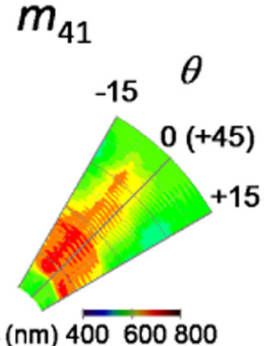

e

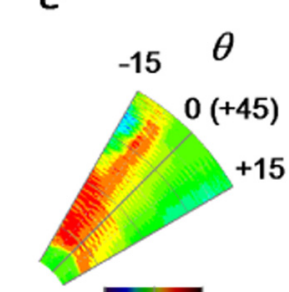

$\lambda(\mathrm{nm}) \overline{400600800}$
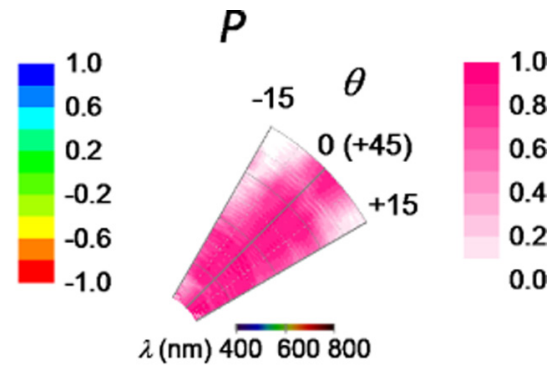

$|\alpha|$
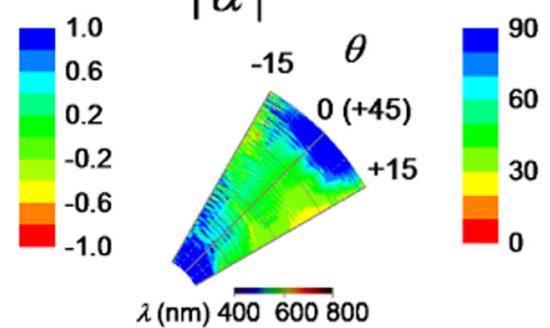

FIG. 7. Scattering contour plots of MMSE data for C. chrysargyrea showing $m_{41}, P, e$, and $|\alpha|$ in the range $\lambda \in[245,1000] \mathrm{nm}$ and $\theta \in\left[45^{\circ} \pm 15^{\circ}\right]$. 

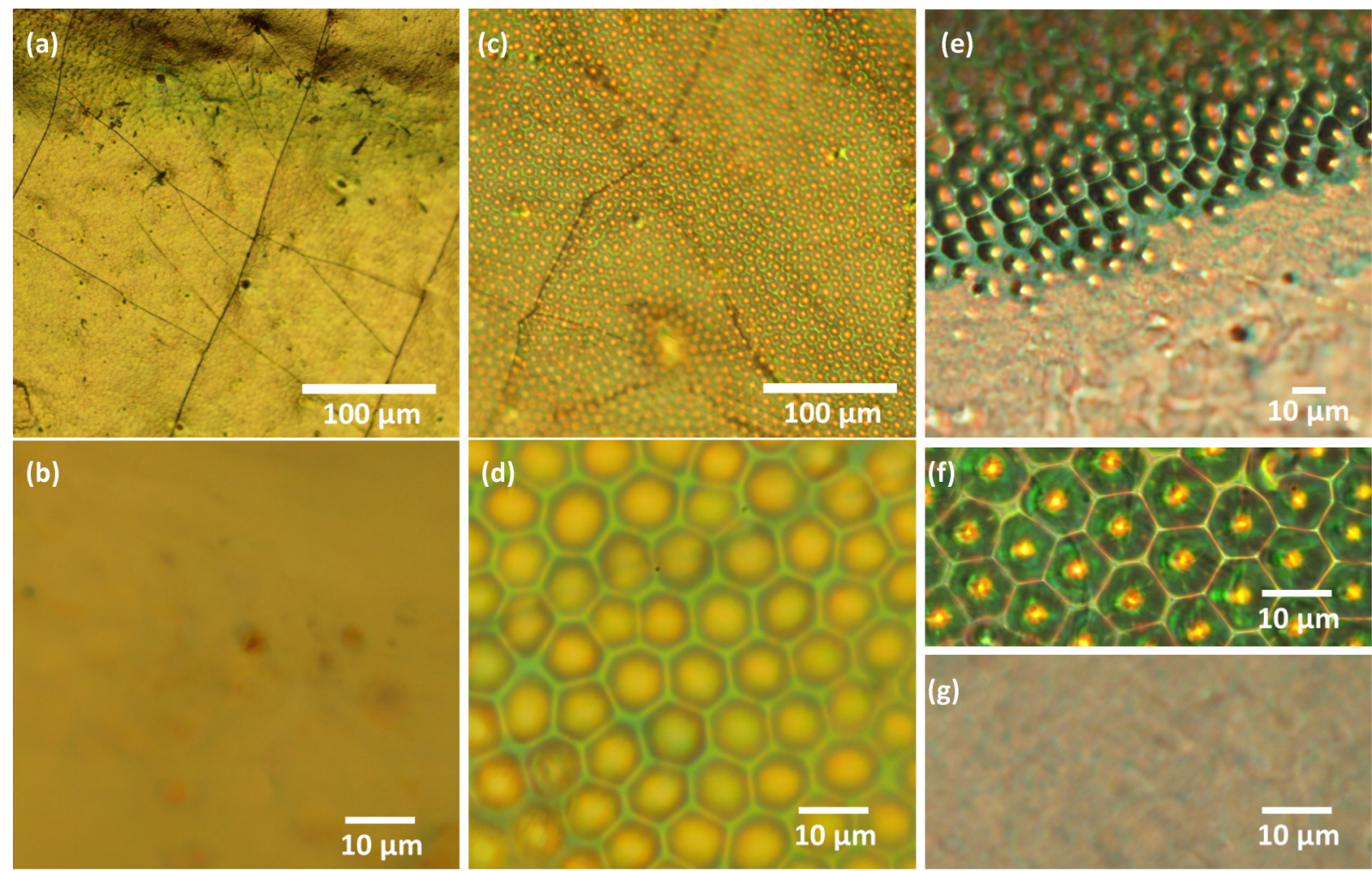

FIG. 8. Optical microscopy images of (a, b) C. chrysargyrea, (c, d) C. woodi, and (e-g) C. gloriosa. The image in (f) is from a green-colored area and $(\mathrm{g})$ from a metallic-colored area.

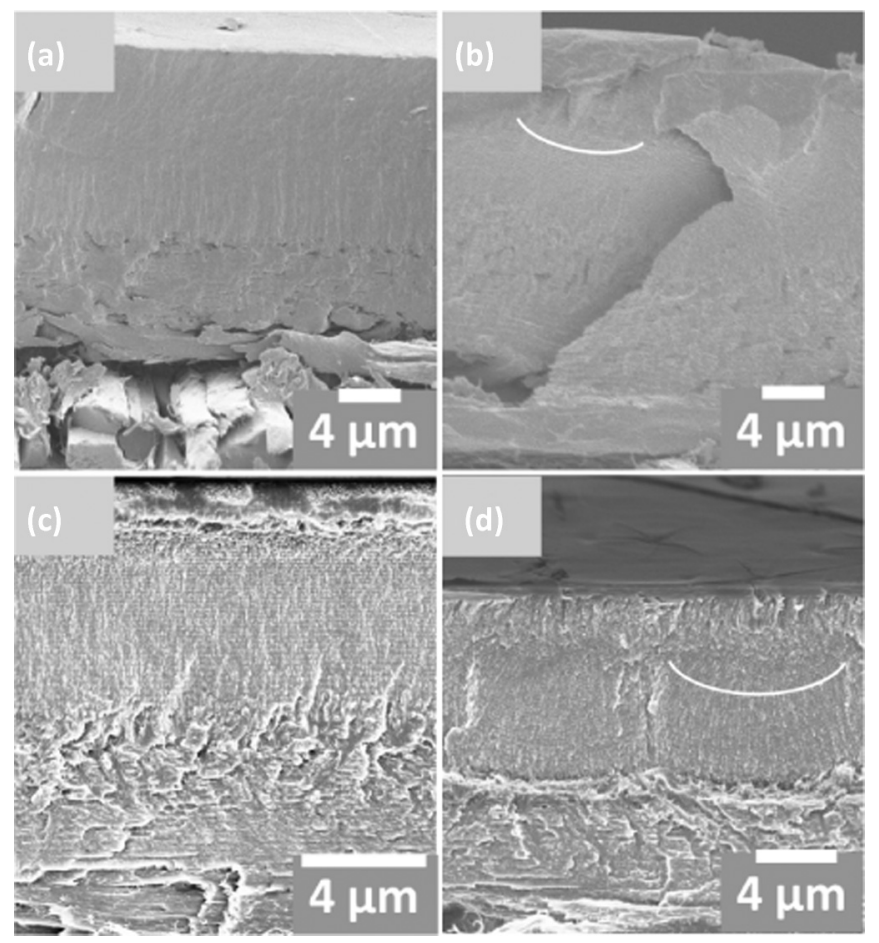

FIG. 9. SEM images of the cross section of (a) C. chrysargyrea, (b) C. woodi, and C. gloriosa (c) on the metallic-colored area and (d) on the green area.
Circular polarization in light reflected from the cuticle is attributed to the presence of Bouligand structures. Such structures are hard to resolve with SEM but have been observed in transmission electron microscopy images [29-31].

\section{Scanning electron microscopy}

The SEM cross-section images of the elytras show two parts in the exocuticle. The outermost part is known as the outer exocuticle and the inner one as the inner exocuticle.

SEM images of the metallic-colored beetle and the goldcolored areas of C. gloriosa in Figs. 9(a) and 9(c), respectively, show similar structures with sublayers parallel to the surface of the exoskeleton. The thickness of the sublayers decreases towards the surface.

The SEM images of the green-colored beetle $C$. wood $i$ in Fig. 9(b) and the green-colored areas of C. gloriosa in Fig. 9(d) show a different structure. The inner exocuticle has a stack of sublayers parallel to the surface. However, in the outer part the sublayers are concentrically curved and form a cusplike structure with a width of around $10 \mu \mathrm{m}$.

\section{CONCLUDING REMARKS}

The MMSE analysis shows that the beetles examined in this study, all belonging to the same genus, can be classified into two groups depending on the type of polarization behavior. One group is exemplified by the green-colored $C$. macropus, 
C. peruviana, and C. woodi that only show ordinary linear polarization effects but very small elliptical polarization effects for specular reflection. The second group is exemplified by those with metallic coloration, C. argenteola, C. resplendens, and $C$. chrysargyrea, that show very large elliptical polarization effects. These differences can be explained with scattering observations that show that the green beetles also show polarization but for off-specularly scattered light. $C$. gloriosa exhibits both types of effects; its green-colored areas belong to the first group and its gold-colored areas to the second.

These results are due to differences in the structure in the exocuticle as confirmed by the optical microscopy and SEM studies. The metallic-colored beetles and gold-colored areas of $C$. gloriosa have a flat surface within the beam spot and the layers in the exocuticle are parallel to this surface. Such a structure acts as a specular reflector. On the other hand, the structure of the exoskeleton of the green beetles and the green areas of $C$. gloriosa show a textured surface with depressions that correspond to the cusplike structure seen in the outer exocuticle. Simulation of cuticle reflection properties has been performed by several groups $[14,15]$, but electromagnetic modeling of beetle cuticle is a complex task and has so far only been performed for simple structures [17].

Chrysina is a genus with around 100 species [32]. Nearly $75 \%$ of the species are green colored and it may be anticipated that they have similar polarization behavior as the green beetles presented in this study. Less than $20 \%$ of the Chrysina species have metallic coloration with different silver-colored or goldcolored tones which may have similar reflection properties as
C. chrysargyrea and the other metallic-colored beetles in this study.

From this point it seems that at least two types of polarization behavior are common in the Chrysina genus but with possibilities that some have other polarization effects. There are, for instance, a few Chrysina beetles having a brownish coloration that may not be included in the classification above. Two of these are the striped $C$. adelaida (included in Table I) and $C$. quetzalcoatli with green stripes similar to C. gloriosa but with the other stripes in a red-brown color instead of metallic. Some initial studies of $C$. adelaida have shown more effects in the near infrared wavelength region, thus indicating a different structure. The minority of Chrysina beetles with brownish coloration will be the subject of a future study.

\section{ACKNOWLEDGMENTS}

Jan Landin, Torun Berlind, and Roger Magnusson are acknowledged for valuable discussions and their assistance with instrumentation. Dr. Parrish Brady, University of Texas at Austin, is acknowledged for providing beetle specimens. The Swedish Museum of Natural History in Stockholm, the National Museum of Natural Science in Madrid, the Natural History Museum in London, and the Museum of Natural Science in Berlin are acknowledged for loan of specimens. Financial support was obtained from the Knut and Alice Wallenberg foundation, the Swedish Research Council, and The Centre in Nano Science and Nano Technology (CeNano) at Linköping University.
[1] A. A. Michelson, On metallic colouring in birds and insects, Philos. Mag. 21, 554 (1911).

[2] L. D. Silva, I. Hodgkinson, P. Murray, Q. H. Wu, M. Arnold, J. Leader, and A. McNaughton, Natural and nanoengineered chiral reflectors: Structural color of manuka beetles and titania coatings, Electromagnetics 25, 391 (2005).

[3] D. J. Brink, N. G. van der Berg, L. C. Prinsloo, and I. J. Hodgkinson, Unusual coloration in scarabaeid beetles, J. Phys. D: Appl. Phys. 40, 2189 (2007).

[4] T. Lenau and M. Barfoed, Colours and metallic sheen in beetle shells. A biomimetic search for material structuring principles causing light interference, Adv. Eng. Mater. 10, 299 (2008).

[5] J. W. Galusha, L. R. Richey, J. S. Gardner, J. N. Cha, and M. H. Bartl, Discovery of a diamond-based photonic crystal structure in beetle scales, Phys. Rev. E 77, 050904 (2008).

[6] A. E. Seago, P. Brady, J.-P. Vigneron, and T. D. Schultz, Gold bugs and beyond: A review of iridescence and structural colour mechanisms in beetles (Coleoptera), J. R. Soc. Interface 6, S165 (2009).

[7] C. Campos-Fernández, D. E. Azofeifa, M. Hernández-Jiménez, A. Ruiz-Ruiz, and W. E. Vargas, Visible light reflection spectra from cuticle layered materials, Opt. Mater. Express 1, 85 (2011).

[8] D. G. Stavenga, Thin film and multilayer optics cause structural colors of many insects and birds, Mater. Today: Proc. 1, 109 (2014).

[9] D. H. Goldstein, Polarization properties of Scarabaeidae, Appl. Opt. 45, 7944 (2006).
[10] R. Hegedüs, G. Szél, and G. Horváth, Imaging polarimetry of the circularly polarizing cuticle of scarab beetles (Coleoptera: Rutelidae, Cetoniidae), Vision Res. 46, 2786 (2006).

[11] S. A. Jewell, P. Vukusic, and N. W. Roberts, Circularly polarized colour reflection from helicoidal structures in the beetle Plusiotis boucardi, New J. Phys. 9, 99 (2007).

[12] S. Lowrey, L. D. Silva, I. Hodgkinson, and J. Leader, Observation and modeling of polarized light from scarab beetles, J. Opt. Soc. Am. A 24, 2418 (2007).

[13] V. Sharma, M. Crne, J. O. Park, and M. Srinivasarao, Structural origin of circularly polarized iridescence in jeweled beetles, Science 325, 449 (2009).

[14] I. Hodgkinson, S. Lowrey, L. Bourke, A. Parker, and M. W. McCall, Mueller-matrix characterization of beetle cuticle: Polarized and unpolarized reflections from representative architectures, Appl. Opt. 49, 4558 (2010).

[15] W. E. Vargas, M. Hernández-Jiménez, E. Libby, D. E. Azofeifa, Á. Solis, and C. Barboza-Aguilar, Broadening of effective photonic band gaps in biological chiral structures: From intrinsic narrow band gaps to broad band reflection spectra, Europhys. Lett. 111, 64001 (2015).

[16] H. Arwin, R. Magnusson, J. Landin, and K. Järrendahl, Chirality-induced polarization effects in the cuticle of scarab beetles: 100 years after Michelson, Philos. Mag. 92, 1583 (2012).

[17] H. Arwin, T. Berlind, B. Johs, and K. Järrendahl, Cuticle structure of the scarab beetle Cetonia aurata analyzed by 
regression analysis of Mueller-matrix ellipsometric data, Opt Express 21, 22645 (2013).

[18] H. Arwin, L. Fernández Del Río, and K. Järrendahl, Comparison and analysis of Mueller-matrix spectra from exoskeletons of blue, green and red Cetonia aurata, Thin Solid Films 571, 739 (2014).

[19] H. Arwin, R. Magnusson, L. Fernández del Río, C. Åkerlind, E. Muñoz Pineda, J. Landin, A. Mendoza-Galván, and K. Järrendahl, Exploring optics of beetle cuticles with Muellermatrix ellipsometry, Mater. Today: Proc. 1, 155 (2014).

[20] L. Fernández del Río, H. Arwin, and K. Järrendahl, Polarizing properties and structural characteristics of the cuticle of the scarab beetle Chrysina gloriosa, Thin Solid Films 571, 410 (2014).

[21] K. Järrendahl and H. Arwin, Ellipsometry of Functional Organic Surfaces and Films, edited by K. J. Eichhorn and K. Hinrichs (Springer-Verlag, Berlin, 2013).

[22] V. Sharma, M. Crne, J. O. Park, and M. Srinivasarao, Bouligand structures underlie circularly polarized iridescence of scarab beetles: A closer view, Mater. Today: Proc. 1, 161 (2014).

[23] L. T. McDonald, E. D. Finlayson, and P. Vukusic, Untwisting the polarization properties of light reflected by scarab beetles, Proc. SPIE 9341, 93410K (2015).

[24] P. Brady and M. Cummings, Differential response to circularly polarized light by the jewel scarab beetle Chrysina gloriosa, Am. Nat. 175, 614 (2010).
[25] M. Blahó, A. Egri, R. Hegedüs, J. Jósvai, M. Tóth, K. Kertész, L. P. Biró, G. Kriska, and G. Horváth, No evidence for behavioral responses to circularly polarized light in four scarab beetle species with circularly polarizing exocuticle, Physiol. Behav. 105, 1067 (2012).

[26] A. Mendoza-Galván, E. Muñoz Pineda, K. Järrendahl, and H. Arwin, Evidence for a dispersion relation of optical modes in the cuticle of the scarab beetle Cotinis mutabilis, Opt. Mater. Express 4, 2484 (2014).

[27] H. Fujiwara, Spectroscopic Ellipsometry: Principles and Applications (Wiley, New York, 2007).

[28] E. Collett, Polarized Light: Fundamentals and Applications (Marcel Dekker, New York, 1993).

[29] A. C. Neville, Biology of the Arthropod Cuticle (SpringerVerlag, Berlin, 1975).

[30] M. Locke, The cuticle and wax secretion in Calpodes ethlius (Lepidoptera, Hesperidae), J. Cell Sci. s3-101, 333 (1960).

[31] A. C. Neville and B. M. Luke, A two-system model for chitin-protein complexes in insect cuticles, Tissue Cell 1, 689 (1969).

[32] Division of Entomology, University of Nebraska, Generic guide to new world scarab beetles, http://www.museum.unl. edu/research/entomology/guide/guide-introduction/guideintro. html 